\title{
Simultaneous Determination of Ionic Liquid Cations and Anions Using Ion Chromatography with Tandem Ion Exchange Columns: a Preliminary Assessment
}

\author{
Aleksandra MARKowsKa and Piotr StePnOwSKi ${ }^{\dagger}$ \\ Department of Environmental Analysis, Faculty of Chemistry, University of Gdańsk, \\ ul. Sobieskiego 18, PL-80-952 Gdańsk, Poland
}

\begin{abstract}
The usefulness of a cation exchange system followed by conductometric detection for alkylimidazolium cations separation and analysis was found for the first time. Furthermore, the possibility of simultaneously separating cations and anions typically used in ionic liquids in one chromatographic run was tested. A tandem of columns containing silica-based strong anion exchange and strong cation exchange packings was used. The study's objective was to find the optimal mobile phase: the best results were obtained with one consisting of potassium hydrogen phthalate and phthalic acid modified with acetonitrile. The method is simple and selective, and is believed to be applicable to numerous ionic liquids based on a similar design.
\end{abstract}

(Received April 15, 2008; Accepted May 28, 2008; Published October 10, 2008)

\section{Introduction}

Room temperature ionic liquids (RTIL) are a new class of compounds usually consisting of nitrogen- or phosphoruscontaining organic cations such as alkylimidazolium, alkylpyridinium, alkylpyrrolidinium or alkylphosphonium combined with a variety of organic or inorganic anions. Apart from their negligible vapor pressure, believed to be the main "green" feature of ionic liquids, one of their most attractive characteristics is the potential to be "designed to order". Liquid below $100^{\circ} \mathrm{C}$, these compounds make ideal non-volatile solvents for a variety of industrial processes such as media for organic synthesis and catalysis, as alternative electrolytes in batteries and solar cells, as phases and phase modifications in the separation sciences, and as alternative lubricants. ${ }^{1}$

Because of their widespread potential application in industrial processes, ionic liquids should also be evaluated with respect to the methods of their determination. So far this has been done through cation analyses in matrices such as solid materials, biological and environmental samples, and aqueous solutions. The relevant analytical techniques are intended to selectively separate the quaternary entity from other cationic and non-ionic species present in the matrix and to enable its fine-scale quantification. Present-day methods of cation analysis are based primarily on high performance liquid chromatography employing several types of chemically modified stationary phases and various buffered mobile phases. ${ }^{2}$ Our group has also recently developed two reliable and accurate methods for the quantitative and qualitative determination of typical ionic liquid anions based on suppressed and non-suppressed ion chromatography followed by conductometric detection. ${ }^{3}$

Regardless of the type of potential analytical matrix, the cations and anions of ionic liquids will always be present together. Most probably such a situation will not involve just one cation and one anion as residues in products or materials that have been in contact with such solvents. Future residual analysis of these compounds will have to take the form of a multi-component analysis capable of dealing with a variety of cations and anions derived from ionic liquid blends, reactor cross contamination or residues in materials and products from ionic liquid technology.

As already mentioned, present-day methods for determining ionic liquid entities analyze cations and anions separately, and in consequence, two different sets of analytical instrumentation and conditions are needed. This state of affairs can be alleviated immediately if the sample is analyzed using one instrument and one set of chromatographic conditions. Such an approach involves the use of a mixed bed of cation and anion exchange materials ${ }^{4,5}$ or, more frequently, cation and anion exchange columns connected in tandem. . $^{6-9}$

It seems that as analytical targets ionic liquids are media tailor-made for this type of analytical approach. In this study, therefore, it was decided to develop, for the first time, an analytical method with the initial aim of simultaneously separating typical ionic liquid cations and anions in a single chromatographic run. The tandem of columns consisted of silica-based strong anion exchange packings and strong cation exchange packings; the research was focused on finding an optimal mobile phase.

The method was developed to gain possible applicability in purity assessment and quality assurance of materials and commercial products in ionic liquid industry as well as in the assessment of potential environmental and food contamination with ionic liquids.

$\dagger$ To whom correspondence should be addressed.

E-mail: sox@chem.univ.gda.pl 


\section{Experimental}

\section{Reagents and chemicals}

The ionic liquids chosen for these studies, 1-butyl-3methylimidazolium (BMIM) chloride, bromide, tetrafluoroborate $\left(\mathrm{BF}_{4}^{-}\right), \quad$ bis(trifluoromethanesulfonyl)imide $\left(\mathrm{Tf}_{2} \mathrm{~N}^{-}\right)$and hexafluorophosphate $\left(\mathrm{PF}_{6}^{-}\right) ;$1-amyl-3-methylimidazolium (AMIM) chloride, 1-propyl-3-methylimidazolium (PMIM) chloride; 1-hexyl-3-methylimidazolium (HMIM) chloride and bis(trifluoromethanesulfonyl)imide; 1-octyl-3-methylimidazolium (OMIM) chloride and tetrafluoroborate, were obtained from Merck (Darmstadt, Germany). In addition, 1-decyl-3methylimidazolium (DMIM) chloride, 1-nonyl-3-methylimidazolium (NMIM) chloride and hexafluorophosphate were obtained from the Ionic Liquids Collection of the working group of Prof. Bernd Jastorff (Center for Environmental Research and Technology, UFT, University of Bremen). Orthophosphoric acid, potassium dihydrogen phosphate, potassium hydrogen phthalate, phthalic acid, 5-sulfosalicylic acid, tris(hydroxymethyl)aminomethane (Tris), all p.a. quality, were purchased from POCh (Gliwice, Poland); benzoic acid was from Fluka, and HPLC grade acetonitrile and methanol from Lab-Scan (Dublin, Ireland). The final mixture of ten ions was prepared in $20 \%$ (v/v) of acetonitrile, which was an optimal solution to prevent the progressive hydrolysis of tetrafluoroborate. ${ }^{10}$

\section{Apparatus}

The high performance liquid chromatography apparatus consisted of an LC 10 AT vp metering pump (Shimadzu Ltd.), with a syringe-loading sample injector and a $100-\mu \mathrm{L}$ sample loop (Rheodyne). The chromatographic experiments were carried out with a silica-based strong anion exchange column (Phenosphere SAX, with quaternary ammonium groups), and a strong cation exchange column (Luna SCX, with sulfonic groups) (Phenomenex), both of length $250 \mathrm{~mm}$, i.d. $=4.6 \mathrm{~mm}$ and particle size $5 \mu \mathrm{m}$. The columns were connected in tandem into one conductometric detector. Detection was achieved with a 732 IC conductivity detector (Metrohm). All chromatographic data were recorded using IC Net 2.3 Metrohm software. Before use, the mobile phase was filtered (filter papers; MachereyNagel, Germany) and degassed in an ultrasonic bath.

\section{Procedure}

In the course of this study, several mobile phase compositions consisting of phosphate buffer or potassium hydrogen phthalate/ phthalic acid or 5-sulfosalicylic and benzoic acids were tested. Additionally, Tris buffer or sodium hydroxide was used to control the $\mathrm{pH}$ of the mobile phase. Acetonitrile was used as organic modifier. In all cases, the mobile phase $\mathrm{pH}$ was measured following the addition of the organic modifier.

\section{Results and Discussion}

At first, mobile phase parameters such as $\mathrm{pH}$, ionic strength and organic modifier content were varied in order to obtain satisfactory separation conditions of all the ions investigated. It is known that in the non-suppressed IC mode followed by conductometric detection, the eluting power of the eluting ion should be relatively high, even at low concentrations. Low concentrations furthermore ensures a background conductivity lower than that of the analyte. To achieve these conditions, researches have recommended polybasic organic acids as eluting ions. ${ }^{11}$ An initial assessment was therefore made of the

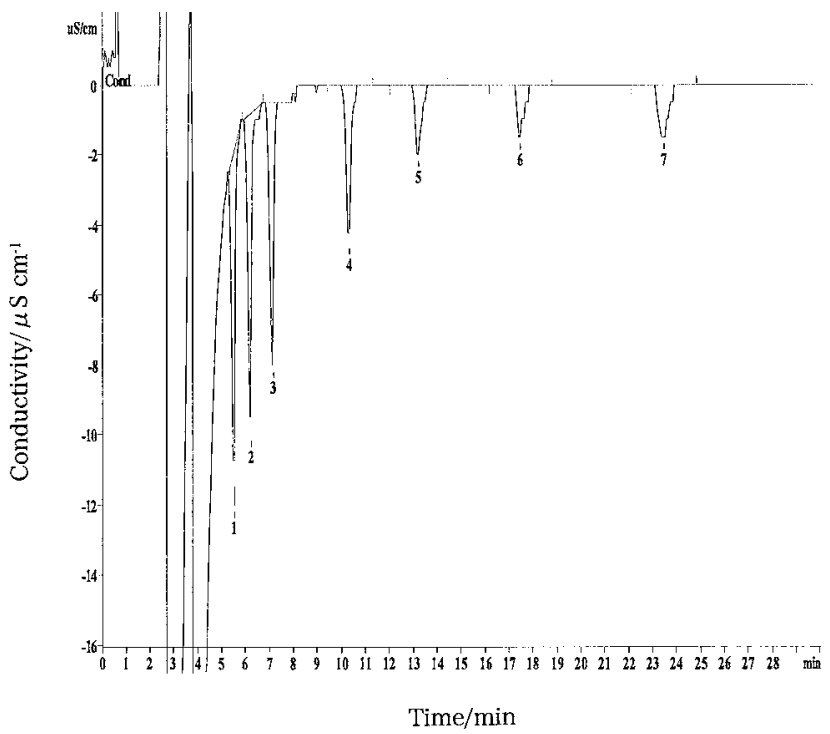

Fig. 1 Separation of seven ionic liquid cations: 1, DMIM; 2, NMIM; 3, OMIM; 4, HMIM; 5, AMIM; 6, BMIM; 7, PMIM (0.5 $\mathrm{mM}$ each). Mobile phase: $18 \mathrm{mM}$ potassium hydrogen phthalate/2 $\mathrm{mM}$ phthalic acid $\mathrm{pH} 4.46 ; 60 \%$ acetonitrile $(\mathrm{v} / \mathrm{v})$. Strong cation exchange column Luna SCX, absolute conductivity of mobile phase, $1239 \mu \mathrm{S} / \mathrm{cm}$; flow rate, $1 \mathrm{~mL} / \mathrm{min}$; injection volume, $100 \mu \mathrm{L}$. Conductivity detection.

usefulness of a phosphate buffer $\left(\mathrm{KH}_{2} \mathrm{PO}_{4} / \mathrm{H}_{3} \mathrm{PO}_{4}\right)$ adjusted to different $\mathrm{pH}$ and modified with acetonitrile in different proportions. This mobile phase system was found useful only for the effective separation of the cations under investigation; the anions remained unseparated, regardless of the composition used. Also tested was the applicability of the frequently used 5sulfosalicylic acid and benzoic acid systems, both adjusted with Tris buffer and sodium hydroxide to the desired $\mathrm{pH}$. In general, both these systems suffered from poor selectivity: only three anions (chloride, bis(trifluoromethanesulfonyl)imide and hexafluorophosphate) could be separated. Moreover, no simultaneous separation of cations was possible with this mobile phase. The number of separated ions increased when the mobile phase $\mathrm{pH}$ was changed. However, this also caused the degree of ionization of the eluting ions to increase significantly, which led to a substantial change in the final background conductivity.

Finally, a phthalate mobile phase was tested; this consisted of potassium hydrogen phthalate and phthalic acid in various proportions mixed with acetonitrile as organic modifier. The optimal mobile phase for alkylimidazolium cation separation consisted of $18 \mathrm{mM}$ potassium hydrogen phthalate and $2 \mathrm{mM}$ phthalic acid ( $\mathrm{pH} 4.46)$ mixed with $60 \%(\mathrm{v} / \mathrm{v})$ of acetonitrile. Not only does this phase provide a suitable elution strength; the risk of buffer crystallization in the HPLC system is minimal, and the background conductivity is acceptable. The chromatogram in Fig. 1 shows the separation of the cations under investigation. The separation of the ionic liquid anions (chloride, bromide, tetrafluoroborate, hexafluorophosphate and bis(trifluoromethanesulfonyl)imide), however, was quite poor under these particular conditions. Therefore, different molar concentration ratios of potassium hydrogen phthalate to phthalic acid were tested in the course of this study. Changing the ratio of the phthalate buffer constituents to $5 \mathrm{mM}$ potassium hydrogen phthalate and $15 \mathrm{mM}$ of phthalic acid, and the proportion of organic modifier to $45 \%(\mathrm{v} / \mathrm{v})$, additionally improved the resolution of the anions under investigation. Under such 


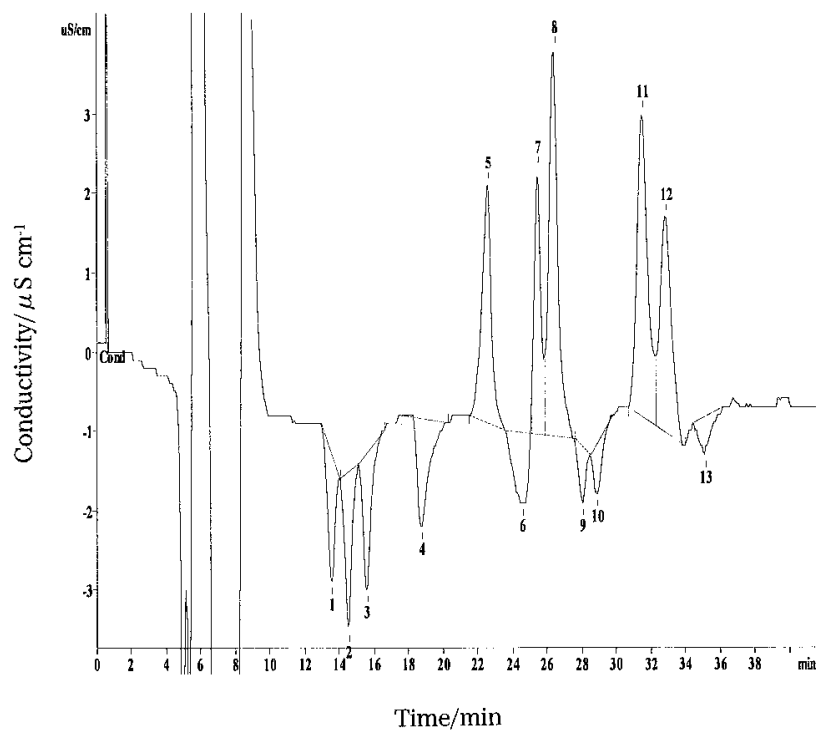

Fig. 2 Separation of a mixture of ionic liquid cations and anions (concentration $0.5 \mathrm{mM}$ each) with use of tandem ion exchange columns. 1, DMIM; 2, NMIM; 3, OMIM; 4, HMIM; 5, $\mathrm{Tf}_{2} \mathrm{~N}^{-} ; 6$, AMIM; 7, $\mathrm{PF}_{6}^{-} ; 8, \mathrm{BF}_{4}^{-} ; 11, \mathrm{Br}^{-} ; 12, \mathrm{Cl}^{-} ; 13, \mathrm{BMIM}$; signals 9,10 unidentified. Conductivity detection. Mobile phase: $5 \mathrm{mM}$ potassium hydrogen phthalate/15 mM phthalic acid, $45 \%(\mathrm{v} / \mathrm{v})$ acetonitrile $\mathrm{pH}$ 3.05. Absolute conductivity of mobile phase, $476 \mu \mathrm{S} / \mathrm{cm}$; flow rate, 1 $\mathrm{mL} / \mathrm{min}$; injection volume, $100 \mu \mathrm{L}$. Columns in the order: strong anion exchange Phenosphere SAX, strong cation exchange Luna SCX

conditions, therefore, it was possible to separate in one chromatographic run cations and anions on tandem ion exchange columns followed by conductometric detection. Figure 2 illustrates the separation of ten ions: $\mathrm{Cl}^{-}, \mathrm{Br}^{-}, \mathrm{Tf}_{2} \mathrm{~N}^{-}, \mathrm{BF}_{4}^{-}, \mathrm{PF}_{6}^{-}$, 1- $n$-decyl-3-methylimidazolium, 1-methyl-3- $n$-nonylimidazolium, 1-methyl-3-n-octylimidazolium, 1-hexyl-3-methylimidazolium and 1-n-butyl-3-methylimidazolium. In this example, they are separated using first anion and then cation exchange columns in tandem. Changing the order of the columns does not significantly affect the quality of separation.

Validation parameters such as detection (LOD) and quantitation limits (LOQ), repetitively linear dynamic range and repeatability of the method were also estimated (Table 1). The specific baseline enables reliable integration of all the signals Very good calibration curves with excellent correlation coefficients suggest that this method enables reliable quantitative analyses. Small differences in linear ranges among analyzed ions are caused by wavy structures of baselines, repeatable enough however to keep very good analytical parameters. The LOD values are in the range from 0.008 to $0.050 \mathrm{mM}$; they are much lower in the case of anions if compared to organic cations.

Literature data reporting on limits of detection of simple inorganic anions and cations in tandem ion chromatography range between 0.00015 and $0.004 \mathrm{mM} .^{12}$ In the case of capillary electrophoresis, also used for the simultaneous determination of anions and cations, the minimum detectable levels ranged from $0.2-1.9 \mathrm{mg} / \mathrm{L}$ and are comparable with ion chromatographic methods. ${ }^{13}$

\section{Conclusions}

The paper reports the first routine chromatographic method to
Table 1 Analytical performance parameters

\begin{tabular}{lcccc}
\hline \multicolumn{1}{c}{ Ion } & $\begin{array}{c}\text { Repeatability, } \\
\mathrm{RSD}^{\mathrm{a}}\end{array}$ & $\begin{array}{c}\text { Linearity, } \\
R^{\mathrm{b}}\end{array}$ & LOD/mM & LOQ/mM \\
\hline $\mathrm{Cl}^{-}$ & 0.0309 & 0.999 & 0.008 & 0.016 \\
$\mathrm{Br}^{-}$ & 0.0258 & 0.997 & 0.008 & 0.016 \\
$\mathrm{Tf}_{2} \mathrm{~N}^{-}$ & 0.0192 & 0.998 & 0.008 & 0.016 \\
$\mathrm{BF}_{4}{ }^{-}$ & 0.0146 & 0.999 & 0.009 & 0.018 \\
$\mathrm{PF}_{6}$ & 0.0313 & 0.998 & 0.009 & 0.018 \\
$\mathrm{DMIM}$ & 0.0454 & 0.999 & 0.017 & 0.034 \\
$\mathrm{NMIM}$ & 0.0316 & 0.999 & 0.017 & 0.034 \\
OMIM & 0.0720 & 0.999 & 0.017 & 0.034 \\
HMIM & 0.0984 & 0.998 & 0.035 & 0.070 \\
BMIM & 0.1065 & 0.999 & 0.050 & 0.100 \\
\hline
\end{tabular}

a. The short-term repeatability was measured for five injections of the mixture for one concentration $(0.25 \mathrm{mM})$ and is expressed as relative standard deviation (RSD).

b. Linearity range: all anions, $0.02-1 \mathrm{mM}$; DMIM, NMIM and OMIM, 0.02 - $1 \mathrm{mM}$; HMIM and BMIM, $0.05-1 \mathrm{mM}$.

c. Limit of detection was measured for ten sample blanks, each fortified once at lowest acceptable concentration LOD $=0+3 s$ (where $s$ is standard deviation); $\mathrm{LOQ}=2 \times \mathrm{LOD}$.

simultaneously analyze cations and anions as used in ionic liquids, regardless of the sequence of ion exchange columns. Simple and selective, this method is believed be applicable to numerous ionic liquid cations and anions based on a similar design. Further investigations should be undertaken, in particular, regarding the alternatives to and limitations of different buffer systems so as to enable several congeneric types of ionic liquid cations and anions to be analyzed simultaneously.

\section{Acknowledgements}

Financial support was provided by the Polish Ministry of Research and Higher Education under grants: 2P04G 083 29, 2P04G 118 29, BW 8000-5-0138-8 and DS 8200-4-0085-8.

\section{References}

1. P. Wasserscheid and T. Welton, "Ionic Liquids in Synthesis", 2003, Wiley VGH, Weinheim.

2. P. Stepnowski, Int. J. Mol. Sci., 2006, 7, 417.

3. P. Stepnowski and A. Markowska, Aust. J. Chem., in press.

4. W. Hu, H. Tao, M. Tominaga, A. Miyazaki, and H. Haraguchi, Anal. Chim. Acta, 1994, 299, 249.

5. P. N. Nesterenko, Trends Anal. Chem., 2001, 20, 311.

6. K. J. B. A. Karim, Y.-Y. Jin, and T. Takeuchi, J. Chromatogr., A, 2003, 995, 153.

7. M. Amin, L. W. Lim, and T. Takeuchi, Anal. Bioanal. Chem., 2006, 384, 839.

8. Y. Yokoyama, N. Sawaguchi, and H. Sato, Analyst, 2001, 126, 989

9. T. Sakai, Y. Inoue, Y. Date, T. Aoyama, K. Yoshida, and G. Endo, Appl. Organomet. Chem., 2001, 15, 285.

10. C. Villagran, M. Deetlefs, W. R. Pitner, and Ch. Hardacre, Anal. Chem., 2004, 76, 2118.

11. H. Watanabe, Y. Yokoyama, and H. Sato, J. Chromatogr., A, 1996, 727, 311.

12. I. Isildak and A. K. Covington, Electroanalysis, 1993, 5, 815.

13. T. Soga and G. A. Ross, J. Chromatogr., A, 1999, 834, 65. 